\title{
58. A Clinical Experience of Microglioma
}

\author{
Mitsuo Toyama, Masakazu Ikeda, Seishi Fukuma \\ 1st Department of Surgery, Kyoto Medical College \\ and Shizuo MiYake \\ 1st Department of Pathology, Kyoto Medical College
}

Y. K., a man aged 31 years, was admitted to the hospital on Decembe 14, 1963, complaining of headache and nominal aphasia. He had a histor: of mild cerebral concussion one year ago, otherwise had been well until . months previuosly when he had developed high fever up to $38.5^{\circ} \mathrm{C}$ accom panied by left temporal headache with nausea and vomiting lasted for : week. Consequently, the headache persisted and spread to left frontal th occipital area and noticed the difficulty of finding appropriate words fo objects and ideas about 3 weeks prior to the admission. He has complainec of diplopia for the past 2 weeks. Neurological examination upon admissiot revealed bilateral papilloedema, sensory aphasia and mild right sided hemi paresis. The cerebral angiograms were compatible with left temporal lobr tumor. On December 23, 1963, craniotomy was performed to remove entire tumor and adjacent brain tissue in the anterior half of the temporal lobe His post-operative course was uneventful.

The tumor was well circumscribed, pinkish gray in color, rubbery in consistency and a tennis ball in size.

Microscopically, massive necrosis was seen in the central part, the celk were oval or eliptical in the peripheral zone and characteristic perivascular cuffing was seen. The nucleus was oval or eliptical with one or severa] nucleoli and scanty in chromatin. Increased reticulin was found by Pap's reticular stain, particularly in the pericapillary space. The tumor cells intermingled to the reticulin fibers, but direct relation to the tumor cells was obscure. No neurofibrile was stained by Mallory's PTA. Rio-Hortega's microglial stain was in failure. The point of classifing this tumor to microglioma was discussed. Postoperatively, Co-therapy, $3500 \mathrm{r}$ of local doses was given, and he was discharged from the hospital on February 13, 1964 with no neurological deficit. He is still under our follow up, but shows no signs of recurrence what so ever. 